\title{
Detrimental adsorbate fields in experiments with cold Rydberg gases near surfaces
}

\author{
H. Hattermann M. Mack, F. Karlewski, F. Jessen, D. Cano, and J. Fortágh \\ $C Q$ Center for Collective Quantum Phenomena and their Applications, \\ Physikalisches Institut, Eberhard-Karls-Universität Tübingen, \\ Auf der Morgenstelle 14, D-72076 Tübingen, Germany
}

\begin{abstract}
We observe the shift of Rydberg levels of rubidium close to a copper surface when atomic clouds are repeatedly deposited on it. We measure transition frequencies of rubidium to $S$ and $D$ Rydberg states with principal quantum numbers $n$ between 31 and 48 using the technique of electromagnetically induced transparency. The spectroscopic measurement shows a strong increase of electric fields towards the surface that evolves with the deposition of atoms. Starting with a clean surface, we measure the evolution of electrostatic fields in the range between 30 and $300 \mu \mathrm{m}$ from the surface. We find that after the deposition of a few hundred atomic clouds, each containing $\sim 10^{6}$ atoms, the field of adsorbates reaches $1 \mathrm{~V} / \mathrm{cm}$ for a distance of $30 \mu \mathrm{m}$ from the surface. This evolution of the electrostatic field sets serious limitations on cavity QED experiments proposed for Rydberg atoms on atom chips.
\end{abstract}

PACS numbers: 32.30.-r, 32.80.Rm, 68.43.-h

\section{RYDBERG ATOMS AT SURFACES}

The large electric polarizability of Rydberg atoms leads to a large response to electric fields [1]. This property is an enormous advantage for applications that require fast coupling between atoms and photons, such as the entanglement of Rydberg atoms via the electromagnetic modes of radio-frequency and microwave cavities 2. Several quantum computation schemes have been proposed based on Rydberg atoms coupled to superconducting coplanar cavities [3 5]. In the proposed scenarios, cold atomic gases are first positioned near a coplanar resonator. The atoms are subsequently laser excited into Rydberg states which interact with the electromagnetic modes of the resonator. Recent progress with coupling such cavities to superconducting qubits [6, 7] and the coupling of Rydberg atoms to a microwave stripline [8] outline good perspectives.

However, the technical realization faces challenges. One significant problem is the detrimental effect of the electrostatic fields generated by adsorbed atoms on the chip surface. Because of the high electronegativity of metals, atoms deposited on the chip surface partially donate their valence electron to the metal. The result is a permanent electric dipole layer on the surface that produces inhomogeneous electrostatic fields and alters both the energy and orbital structure of nearby Rydberg atoms. The fields can be strong enough to shift Rydberg states out of the cavity resonance.

New chips are initially free of adsorbates, but experiments progressively accumulate adatoms on the surface. An important question is how long it takes until the accumulation of atoms on the surface becomes detrimental. Two research groups reported previously on this subject.

* hattermann@pit.physik.uni-tuebingen.de

$\dagger$ fortagh@uni-tuebingen.de
First, McGuirk et al. and Obrecht et al. studied the electrostatic field of adsorbed atoms on both conducting and insulating surfaces [9, 10]. Second, Tauschinsky et al. measured electrostatic fields of adsorbates using electromagnetically induced transparency (EIT) on Rydberg states 11. The results presented in this article complement the data published by these two groups. We measure the evolution of electrostatic fields at distances of $30-300 \mu \mathrm{m}$ to the surface during a series of consecutive experiments. Starting with a clean copper surface we deposit clouds of ${ }^{87} \mathrm{Rb}$ atoms onto the surface and measure the inhomogeneous electrostatic field of polarized adatoms by spectroscopy on Rydberg states. We find that the electrostatic fields are already significant after about a few hundred experimental cycles. This corresponds to only a few hours of operation for a typical cold atom experiment.

\section{MEASUREMENT OF THE ELECTROSTATIC FIELDS OF ADSORBATES BY RYDBERG EIT}

We measure the electrostatic field of adsorbed and polarized adatoms through the energy shift (DC Stark shift) induced on highly excited Rydberg states of rubidium. We start our experiments with a clean copper surface which is horizontally aligned inside a vacuum chamber (Fig. 1(a)) at a base pressure of $10^{-11}$ mbar. We transport ultracold clouds of ${ }^{87} \mathrm{Rb}(\mathrm{T}=1.5 \mu \mathrm{K})$ with optical tweezers to a position $200 \mu \mathrm{m}$ above the surface and release the atomic cloud. About $\sim 10^{6}$ atoms are dropped in each experimental cycle onto the surface. While the atomic cloud is falling towards the surface, we measure its EIT spectra.

The ladder-type excitation scheme used for the EIT measurements [12, 13] is shown in Fig. 11(b). We probe the absorption on the $5 S-5 P$ transition with a weak probe laser. The $5 P$ state is strongly coupled to a highly excited $n S$ or $n D$ Rydberg state by means of a $480 \mathrm{~nm}$ 
a)

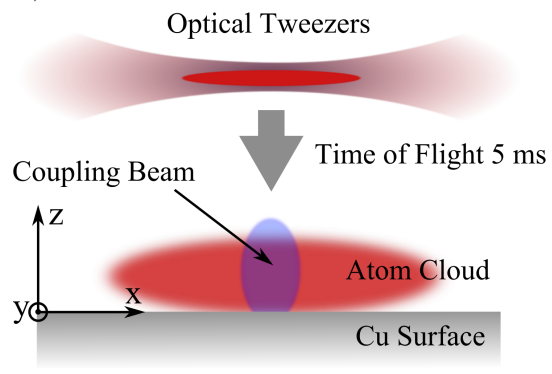

b)

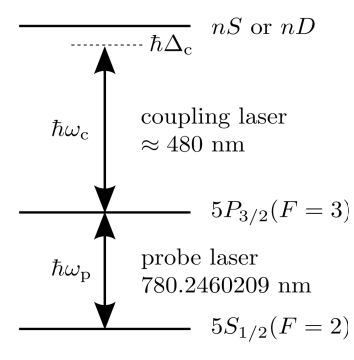

tanouosly focussed onto the cloud. It has an elliptical profile with $\sim 200 \mu \mathrm{m}$ and $300 \mu \mathrm{m}$ FWHM, respectively. The frequency stabilization of the lasers with an absolute accuracy of better than $\pm 1.5 \mathrm{MHz}$ is described in [15].

The measurements are illustrated in Fig. 2(a). The absorption image of an atomic cloud during free fall shows a 'transparency window' at the positions where the coupling laser is resonant with the transition, in this case the $35 D_{5 / 2}\left(m_{J}=1 / 2\right)$ state. The image shows that the resonance condition is satisfied only in a small window revealing a spatial inhomogeneity of the energy shift. In this window the shift of the Rydberg level equals the detuning $\Delta_{C}$ of the coupling laser, in this example $+10 \mathrm{MHz}$ with respect to the unperturbed transition frequency. If the detuning of the coupling laser is changed, the transparency window appears at a different distance from the surface.

We take a series of absorption images as a function of the detuning $\Delta_{C}$. The laser frequency is varied in steps of $1 \mathrm{MHz}$ between consecutive measurements. The results are summarized in Fig. 2(b), which shows the Stark shift of Rydberg states as a function of the distance $z$ to the surface. Each horizontal line of Fig. 2(b) is obtained from the vertical column of absorption images, as indicated by the vertical dashed line in Fig. 2(a). Thus it shows the position of the transparency window for different detunings. The three branches in Fig. 2(b) correspond to the projections of the total angular momentum $J$ of the $35 D_{5 / 2}$ Rydberg state: $\left|m_{J}\right|=1 / 2,3 / 2,5 / 2$.

We now determine the electrostatic field above the copper surface using the measured Stark shifts and comparing them with the theoretically calculated shift of Rydberg levels in electrostatic fields. We calculate the Stark maps with the numerical method of Ref. 17. For our evaluation, we use an algorithm which identifies the electrostatic fields that best fit the measured data. As shown in Fig. 2(c), the results for the three different $\left|m_{J}\right|$ states lie on the same curve, confirming the validity of our procedure. The shifts of the Rydberg states are thus explained by static electric fields alone. The decay of the electrostatic field is modelled here with an exponential function: $E(z)=E_{0} \exp (-z / \sigma)+E_{\text {res }}$, where $z$ is the distance to the surface, $\sigma$ is the decay length and $E_{\text {res }}$ is a residual, homogeneous electrostatic field that accounts for possible external field sources. Figure 2(c) shows the best-fit fields calculated with the three experimental curves of Fig. 2(b). We repeated our measurements on $S$ and $D$ Rydberg states with principal quantum numbers $n$ between 31 and 48 that reproduce the same behaviour. For distances smaller than $30 \mu \mathrm{m}$, the electrostatic field cannot be determined reliably, as high field gradients over the size of one pixel of the camera $(5.6 \mu \mathrm{m}$ in the object plane) lead to blurring of the measured line shifts. We note that the electric field is also inhomogeneous along the $x$-axis. This is a result of the Gaussian distribution of the atomic clouds dropped onto the surface and of the residual roughness of the copper. In order to facilitate the evaluation of the changes of the field with time, all 

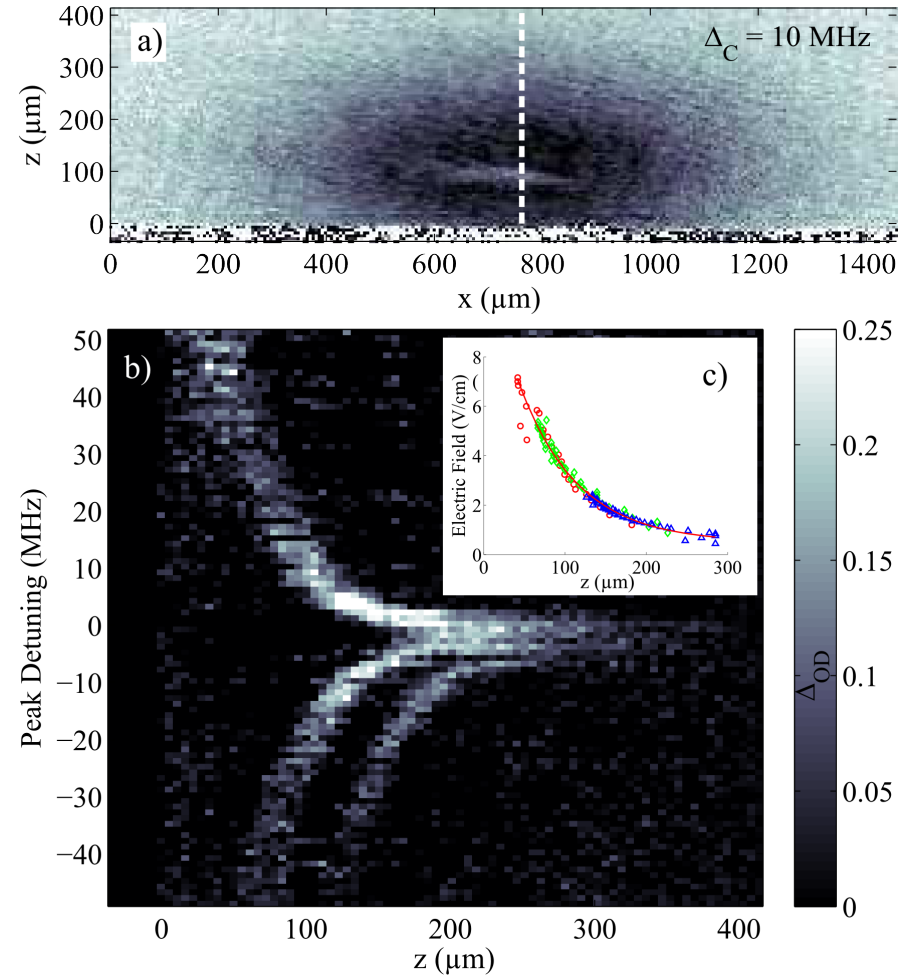

FIG. 2. (a) Absorption image of an atomic cloud during free fall onto the surface taken with the probe beam. The stronger the absorption, the darker the pixel in the image. In this example, the coupling laser is blue detuned by $10 \mathrm{MHz}$ from the $35 D_{5 / 2}$ state of ${ }^{87} \mathrm{Rb}$ and produces a transparency window. The lateral extension of the window is given here by the width of the coupling laser beam. For the data analysis we take the $z$-position of the transparency window along the vertical dashed line. Similar images have been taken for detunings ranging from $-50 \mathrm{MHz}$ to $50 \mathrm{MHz}$, allowing the measurement of electrostatic fields as a function of $z$. (b) Map of the relative optical density as a function of distance to the surface (horizontal axis) and detuning of the coupling laser from the unperturbed transition frequency $5 P_{3 / 2}\left(F=3, m_{F}=3\right) \rightarrow 35 D_{5 / 2}$ (vertical axis). Each horizontal line shows a single measurement. (c) Calculated electrostatic field as function of $z$ using the EIT measurements on the $35 D_{5 / 2}$ state. The different colors are the field strengths obtained using the $\left|m_{J}\right|=1 / 2$ (red circles), 3/2 (green diamonds), and $5 / 2$ states (blue triangles). The data of the three states follow the same curve, which is well approximated by an exponential decay.

the measurements have been evaluated along the same line, as indicated in Fig. 2(a).

\section{TEMPORAL EVOLUTION OF THE ELECTROSTATIC FIELDS OF DEPOSITED ADATOMS}

We evaluate the evolution of the electrostatic field close to the surface as atom clouds are repeatedly deposited on it. Figure 3 summarizes the results. The diagram shows the electric field as a function of the distance from

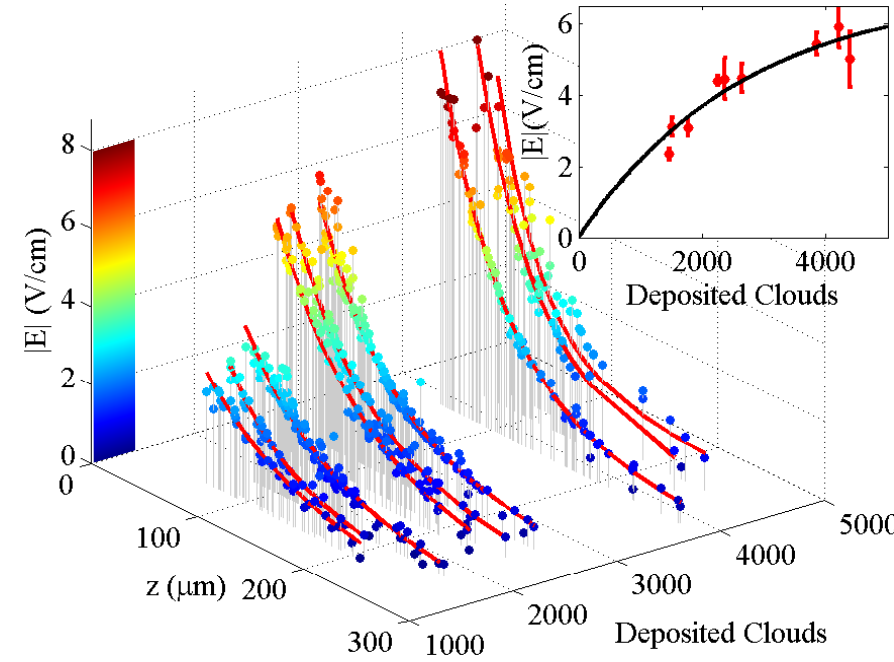

FIG. 3. Measured electrostatic field as a function of $z$ and the number of experimental cycles carried out. Inset: Measured electrostatic field at a distance of $80 \mu \mathrm{m}$ from the surface. We observe an increase of the electric field due to adsorption of Rubidium onto the copper surface. The saturation builds up after the deposition of few thousand atomic clouds.

the surface and the number of deposited atomic clouds. Different colors correspond to different strengths of the electric field. The red lines are exponential fits that we use for determining the electric field as in Sec. II. The inset shows the increase of the measured electric field with the number of deposited atoms for a distance of $80 \mu \mathrm{m}$ from the surface. The magnitude of the field increases with the number of experimental runs. However, we observe a saturation after about ten days of experiments during which we released approximately $5 \cdot 10^{9}$ atoms on the copper surface. Based on our measurements, we estimate that it takes as little as few hundred experimental runs to produce an electric field of $1 \mathrm{~V} / \mathrm{cm}$ at a distance of $30 \mu \mathrm{m}$, assuming a zero-field at the beginning of the experiments. This field produces a level shift of $-2 \mathrm{MHz}$ on the $35 S_{1 / 2}$ and of $-49 \mathrm{MHz}$ on the $55 S_{1 / 2}$ state. This is much larger than the linewidth of a high-Q stripline resonator, making cavity-QED experiments problematic by shifting the atoms out of the cavity resonance.

\section{CONCLUSION}

Our measurements show that neutral atoms adsorbed on a metal surface cause electrostatic fields on the order of $1 \mathrm{~V} / \mathrm{cm}$ after as little as a hundred repetitions of a cold atom experimental cycle. Adsorbate fields have also been observed on dielectric surfaces 18. This sets serious limitations on the feasibility of cavity QED experiments with Rydberg atoms and coplanar cavities. Also dispersion forces between Rydberg atoms and planar surfaces [19] are masked by the strong electric fields of adsorbates. A search for strategies to correct for this problem 
is therefore very important for atom chips. A possible solution could be the cleaning of the surface whenever the electrostatic fields due to adsorbates become harmful. For example, regular heating of the surface cause adsorbed atoms to diffuse. Another possibility would be photodissorption of the adsorbed atoms, but given the work function of metals, this would require light in the far ultraviolet range. Given the fast appearance of detrimental adsorbate fields, an open question is still if there are cleaning techniques which can be applied quickly between experimental cycles. A workaround for this problem would be the development of experimental techniques that avoid deposition of atoms onto the surface or using surface coatings with materials on which no adsorbate fields have been observed 13 . While atoms on surfaces have undesired effects on cold atom experiments, it is worth mentioning that adatoms may be useful to control electric properties of surface layers. For example, alkali metal adsorbates have been used to engineer the electronic structure of graphene [20, 21].
Rydberg EIT can be used for a sensitive measurement of electric fields. In combination with micropositioning of atomic clouds by optical tweezers or magnetic conveyor belts in a scanning probe configuration 22 three dimensional imaging of the electric field distribution is feasible. However, the measurement technique contaminates the surface, which must be taken into account.

\section{ACKNOWLEDGEMENTS}

The authors would like to thank Thomas Judd for useful discussions. This work was supported by the European Research Council (Socathes) and the Deutsche Forschungsgemeinschaft (SFB TRR21). The authors acknowledge additional support from the ev. Studienstiftung Villigst e.V., and the Baden-WürttembergStiftung through the "Kompetenznetz Funktionelle Nanostrukturen".
[1] T. F. Gallagher, Rydberg Atoms (Cambridge University Press, 1994).

[2] J. M. Raimond, M. Brune, and S. Haroche, Rev. Mod. Phys. 73, 565 (2001)

[3] D. Petrosyan and M. Fleischhauer, Phys. Rev. Lett. 100, $170501(2008)$

[4] D. Petrosyan, G. Bensky, G. Kurizki, I. Mazets, J. Majer, and J. Schmiedmayer, Phys. Rev. A 79, 040304 (2009)

[5] M. Saffman, T. G. Walker, and K. Mølmer, Rev. Mod. Phys. 82, 2313 (2010).

[6] L. DiCarlo, J. M. Chow, J. M. Gambetta, L. S. Bishop, B. R. Johnson, D. I. Schuster, J. Majer, A. Blais, L. Frunzio, S. M. Girvin, and R. J. Schoelkopf, Nature 460, 240 (2009)

[7] A. Wallraff, D. Schuster, A. Blais, L. Frunzio, R. Huang, J. Majer, S. Kumar, S. Girvin, and R. Schoelkopf, Nature 431, 162 (2004).

[8] S. D. Hogan, J. A. Agner, F. Merkt, T. Thiele, S. Filipp, and A. Wallraff, Phys. Rev. Lett. 108, 063004 (2012)

[9] J. M. McGuirk, D. M. Harber, J. M. Obrecht, and E. A. Cornell, Phys. Rev. A 69, 062905 (2004)

[10] J. M. Obrecht, R. J. Wild, and E. A. Cornell, Phys. Rev. A 75, 062903 (2007)

[11] A. Tauschinsky, R. M. T. Thijssen, S. Whitlock, H. B. van Linden van den Heuvell, and R. J. C. Spreeuw, Phys.
Rev. A 81, 063411 (2010)

[12] A. K. Mohapatra, T. R. Jackson, and C. S. Adams, Phys. Rev. Lett. 98, 113003 (2007).

[13] H. Kübler, J. P. Shaffer, T. Baluktsian, R. Löw, and T. Pfau, Nature Photonics 4, 112 (2010).

[14] M. Fleischhauer, A. Imamoglu, and J. P. Marangos, Rev. Mod. Phys. 77, 633 (2005)

[15] M. Mack, F. Karlewski, H. Hattermann, S. Höckh, F. Jessen, D. Cano, and J. Fortágh, Phys. Rev. A 83, 052515 (2011)

[16] D. Cano, H. Hattermann, B. Kasch, C. Zimmermann, R. Kleiner, D. Koelle, and J. Fortágh, European Physical Journal D 63, 17 (2011)

[17] M. L. Zimmerman, M. G. Littman, M. M. Kash, and D. Kleppner, Phys. Rev. A 20, 2251 (1979)

[18] R. P. Abel, C. Carr, U. Krohn, and C. S. Adams, Phys. Rev. A 84, 023408 (2011).

[19] J. A. Crosse, S. A. Ellingsen, K. Clements, S. Y. Buhmann, and S. Scheel, Phys. Rev. A 82, 010901 (2010).

[20] T. Ohta, A. Bostwick, T. Seyller, K. Horn, and E. Rotenberg, Science 313, 951 (2006)

[21] K.-H. Jin, S.-M. Choi, and S.-H. Jhi, Phys. Rev. B 82, $033414(2010)$

[22] M. Gierling, P. Schneeweiss, G. Visanescu, P. Federsel, M. Häffner, D. P. Kern, T. E. Judd, A. Günther, and J. Fortágh, Nature Nanotechnology 6, 446 (2011). 